\title{
Studies on Neisseria gonorrhoeae cultured in liquid medium*
}

\author{
JULIA ANN EAVES \\ From Sheffield University Medical School
}

SUMMARY Experiments were carried out to determine the minimum amount of inoculum of gonococci that is needed to produce growth in ANM liquid medium. This was found to be less than had previously been thought. Minimum inocula were used to study penicillin sensitivity in liquid medium. This work showed that growth occurred at higher concentrations of penicillin with larger inocula. Evidence was also obtained which suggested that in some circumstances penicillin acts bacteriostatically rather than bacteriocidally. It was also shown that growth of gonococci can be inhibited in liquid medium by the presence of hyperimmune serum, the inhibition being more marked in the presence of fresh complement. This work is continuing in the hope that it may provide a basis for a much-needed serological test for gonorrhoea. This would be especially valuable in chronic assymptomatic infections.

\section{Introduction}

The gonococcus is a delicate organism which has a limited survival on solid medium. A new ANM liquid medium (Hafiz and McEntegart, 1976) which allows gonococci to survive longer was used to study four aspects of the growth of gonococci:

1. The smallest amount of inoculum needed to initiate growth.

2. The penicillin sensitivities in ANM liquid medium compared with those obtained using the plate method of Reyn et al. (1963).

3. The effects of adding penicillinase to cultures inhibited by penicillin.

4. The effect of specific antiserum on growth of gonococci in ANM liquid medium.

\section{Materials and methods}

ORGANISMS

Numbered stock strains that had been stored in liquid nitrogen and thawed were used. They were plated on Difco GC plates as required. Nine were originally obtained as fresh isolates from the Sheffield Special Clinic; the following international reference strains were also used: $\mathbf{K}_{\mathbf{2}}$ (Kellog), III, V, and VII.

\section{*MSSVD Student Prize 1977}

Address for reprints: J. A. Eaves, 1 Shoreham Avenue, Moorgate, Rotherham, Yorkshire

Received for publication 1 June 1977
MEDIA

The solid medium used was Difco GC medium. The ANM liquid medium was made up as described by Hafiz and McEntegart (1976) and distributed in volumes of $10 \mathrm{ml}$ in universal containers. Corn or soluble starch was used and defined supplement (DS) could be added.

\section{CULTURE}

Inocula used in both plate and liquid cultures were $0.1 \mathrm{ml}$ of serial dilutions of a dense suspension (optical density Brown's tube no. 5) of gonococci in phosphate buffered saline (PBS). The total number of organisms was confirmed by using Miles and Misra counts (Miles et al., 1938). Cultures were incubated at $36^{\circ} \mathrm{C}$ and the $\mathrm{CO}_{2}(10 \%)$ and humidity were automatically regulated.

\section{PENICILLINASE}

In studies of penicillin sensitivity in ANM medium an excess of penicillinase was added to all cultures in which penicillin had inhibited growth so as to determine if the penicillin was acting bacteriocidally or bacteriostatically.

\section{PENICILLIN SENSITIVITY}

1. The standard method described by Reyn et al. (1958) was used to determine the sensitivity of strains of gonococci to penicillin. International reference strains were used to produce a Kaber conversion table for correcting the values, as described by Reyn and Bentzon (1965). 
2. Sensitivity in liquid medium - a modification of the plate method was used. Six penicillin dilutions in ANM liquid medium were made by adding stock solutions of penicillin in broth to ANM medium plus soluble starch and DS. Each dilution in the series was inoculated with gonococci at the determined minimum inoculum, two log dilutions above plus one below (making four in all), Table 2.

L-FORMS

Attempts to isolate L-forms were made by filtering ANM cultures and subculturing the deposit on Difco GC plates containing polyvinylpyrrolidone (Lawson and Douglas, 1973).

\section{ANTISER UM}

Hyperimmune rabbit serum with an antibody titre of $1: 120$ at a complement titre of $1: 20$ was produced by inoculating a rabbit with a suspension of gonococci (strain no. 55) for a period of four weeks. Two other rabbits were inoculated in a similar manner but they died before being bled.

This immune serum was used in an experiment designed to study its effect with and without added complement. Two sources of complement were used -Wellcome freeze-dried complement and fresh guinea-pig serum. Serum and both types of complement were millipore-filtered before use.

Dilutions of immune rabbit serum were doubled (from $1: 20$ to $1: 320$ ) in ANM liquid medium plus soluble starch plus DS. Controls and the following series were set up with the addition of $1 \mathrm{ml}$ reconstituted one-fifth freeze-dried complement or $1 \mathrm{ml}$ guinea-pig serum:

Series 1 Control-ANM+soluble starch (SS) + DS.

Series 2 Control-ANM+SS+DS+guinea-pig serum.

Series 3 Doubling dilutions immune serum + $\mathrm{ANM}+\mathrm{SS}+\mathrm{DS}$.

Series 4 Doubling dilutions immune serum + freeze-dried complement in ANM+SS+DS.

Series 5 Doubling dilutions immune serum + guinea-pig serum (complement) in $\mathrm{ANM}+\mathrm{SS}+$ DS.

All bottles were inoculated with $0 \cdot 1 \mathrm{ml}$ of a standard suspension of GC strain 55 in PBS (Brown's tube no. 5). Miles and Misra counts were done on this suspension. Bottles were incubated and subcultured on to plates at 24 and 48 hours.

\section{Results}

DETERMINATION OF THE MINIMUM AMOUNT OF INOCULUM OF GC IN ANM LIQUID MEDIUM Four types of medium (see Table 1) were inoculated with serial doubling dilutions of standard suspen- sions of gonococci: five strains were used. Miles and Misra counts were made to determine the sizes of inocula; the growth after 24 hours was determined in each bottle by subculturing on to plates. Results in Table 1 show that growth occurred with inocula as small as 10 organisms or less per $10 \mathrm{ml}$ tube of ANM liquid medium.

\section{ANTIBIOTIC SENSITIVITY VALUES}

The antibiotic sensitivity values in liquid medium were compared with those from the routine plate method and the possible effects were investigated of penicillinase on cultures apparently inhibited by penicillin in liquid medium.

For each of 13 strains of gonococci the standard plate sensitivity was measured and the value in liquid medium was given by the penicillin concentration which inhibited the growth of a minimum inoculum of gonococci, the growth being assessed at 24 hours by subculturing on to plates. Penicillinase was added to bottles in which there was no growth after 48 hours; these were then subcultured at 72 hours.

Results for six strains are given in Table 2. They show that inoculum size may affect penicillin susceptibility, and in these cultures growth occurred after the addition of penicillinase.

To compare the two methods the values were arranged in rank order (see Table 3); this showed a correlation which appears to be statistically significant.

\section{INHIBITION OF GROWTH OF GONOCOCCI IN} LIQUID MEDIUM BY ANTISERUM

Growth of gonococci in AMN liquid medium was inhibited when hyperimmune serum was added. This was more marked when complement was also included. Two sets of results for strain no. 55 are shown in Table 4.

\section{Discussion}

\section{MINIMUM INOCULUM}

In $65 \%$ of cases growth occurred in $10 \mathrm{ml} \mathrm{ANM}$ liquid medium with fewer than 10 gonococci which is much less than had previously been thought (Hafiz and McEntegart, 1976).

Four different media were used and ANM plus soluble starch plus DS was chosen as the best medium for future work. Significantly, in the cases of two strains only one gonococcus was estimated to have been inoculated and this produced growth. The small amount of inoculum necessary has led to recent attempts to culture blood samples in liquid medium when looking for gonococcal septicaemia. 
Table 1 The growth of different inocula of gonococci in ANM liquid medium

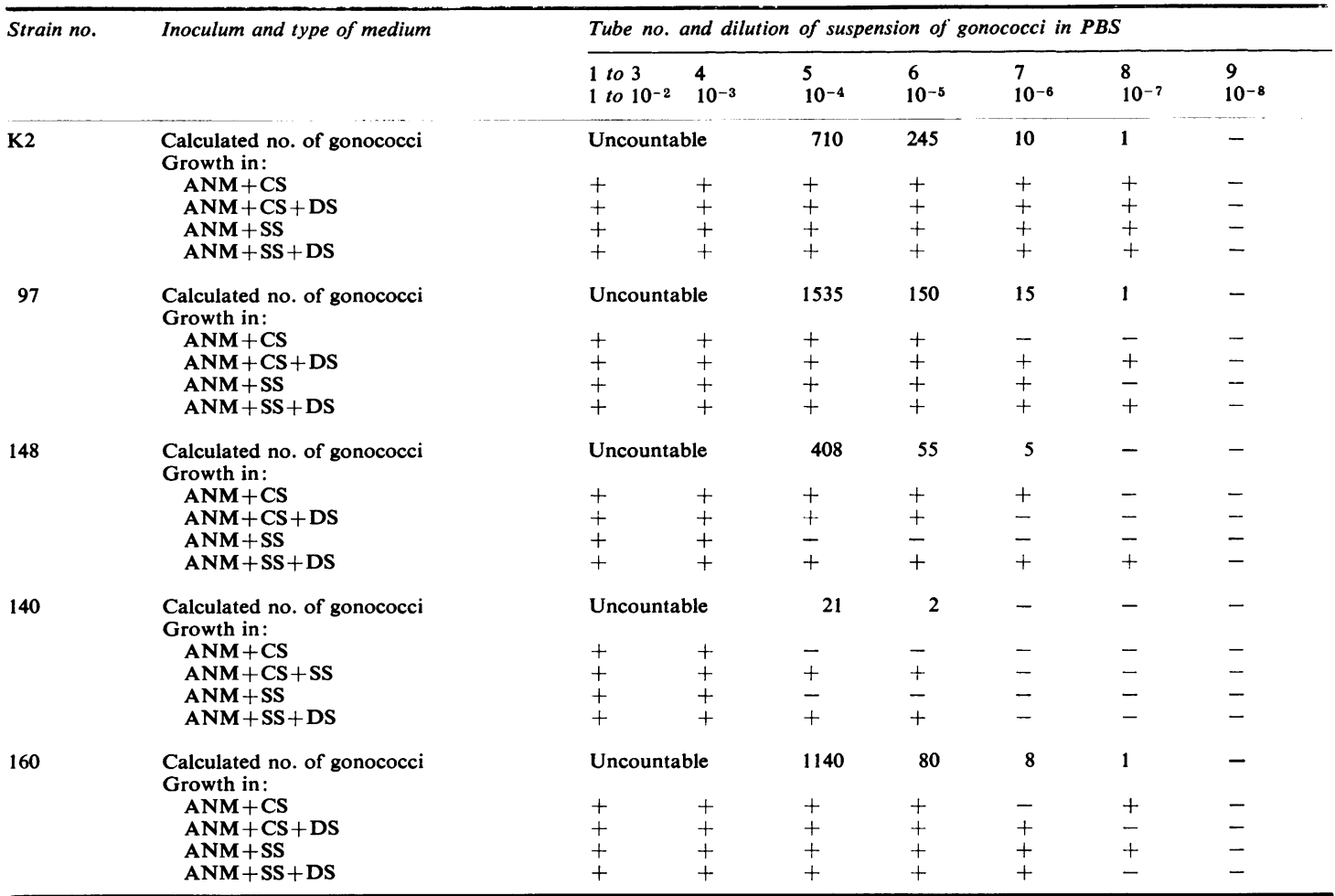

$\mathrm{CS}=$ Corn starch

$\mathrm{SS}=$ Soluble starch

DS $=$ Defined supplement

ANM $=$ ANM liquid medium

+ Growth on subculture at 24 hours

- No growth on subculture at 24 hours

\section{SENSITIVITY OF THE GONOCOCCUS TO}

PENICILLIN

1. There was a correlation between values of penicillin sensitivity obtained by the plate method and by using liquid medium but it is unlikely that liquid cultures will offer any advantage in routine work. This is because (a) contamination is likely to be more troublesome; (b) it is not possible to improve sensitivity; (c) the method is slower, taking 48 rather than 24 hours, and speed is critical in the treatment of gonorrhoea.

2. Inoculum size was shown to have an effect on susceptibility to penicillin. Growth occurred at higher concentrations if a larger inoculum was used. 3 . In three strains out of 13 , growth occurred in cultures only after penicillinase had been added. These were strains $K_{2}, 151$, and 170 . In each case this happened at penicillin concentrations just above the previous limit for growth for each size of inoculum. This result was reproduced for strains 151 and 170.
Penicillin was here apparently behaving bacteriostatically yet it is normally described as bacteriocidal; this may have clinical relevance. It may be related to type as both strains $\mathrm{K}_{2}$ and 151 are colony type 5 producing gonococci. An alternative suggestion is that growth after the addition of penicillinase was caused by reversion of L-forms which had survived in the presence of penicillin; however attempts to substantiate this by demonstrating L-forms using the electron microscope or by culturing filtered samples on plates with incorporated polyvinylpyrrolidone were unsuccessful (Lawson and Douglas, 1973).

INHIBITION OF GROWTH OF GONOCOCCI IN LIQUID MEDIUM BY INCORPORATING SPECIFIC ANTISERUM

Reproducible results were obtained for strain no. 55 . It was shown that by incorporating specific rabbit antiserum growth of gonococci in liquid medium was inhibited, especially when small inocula were 
Table 2 Results of the inhibition of growth of six strains of gonococci in ANM liquid medium by incorporating penicillin. Comparative penicillin sensitivities obtained by the plate method are given

\begin{tabular}{|c|c|c|c|c|c|c|c|c|c|}
\hline \multirow[t]{3}{*}{ Strain no. } & \multirow{3}{*}{$\begin{array}{l}\text { Plate } \\
\text { sensitivity } \\
\text { IC } C_{50} \\
\text { (iu) }\end{array}$} & \multirow{3}{*}{$\begin{array}{l}\text { Corrected } \\
\text { sensitivity } \\
I C_{50} \\
(i u)\end{array}$} & \multirow[t]{3}{*}{$\begin{array}{l}\text { Innoculum } \\
\text { size }\end{array}$} & \multicolumn{6}{|c|}{$\begin{array}{l}\text { Liquid culture } \\
\text { Penicillin concentration in } \mathrm{iu} / \mathrm{ml}\end{array}$} \\
\hline & & & & \multicolumn{6}{|c|}{ Growth on 24-hour subculture } \\
\hline & & & & $1 \cdot 0$ & $\begin{array}{l}2 \\
0 \cdot 25\end{array}$ & $\begin{array}{l}3 \\
0.06\end{array}$ & $\begin{array}{l}4 \\
0.015\end{array}$ & $\begin{array}{l}5 \\
0.004\end{array}$ & $\begin{array}{l}6 \\
\text { Control }\end{array}$ \\
\hline \multirow[t]{3}{*}{ VII } & - & 0.0089 & $17 \times 10^{6}$ & - & - & - & + & + & + \\
\hline & & & 17 & - & - & - & - & + & + \\
\hline & & & 2 & - & - & - & - & + & + \\
\hline \multirow[t]{4}{*}{97} & 1.00 & $1 \cdot 10$ & $94 \times 10^{4}$ & + & + & + & + & + & + \\
\hline & & & $94 \cdot 5$ & - & + & + & + & + & + \\
\hline & & & $9 \cdot 5$ & - & + & + & + & + & + \\
\hline & & & 1.0 & - & + & + & + & + & + \\
\hline \multirow[t]{3}{*}{114} & 0.35 & $8 \cdot 5$ & $23.6 \times 10^{6}$ & - & + & + & + & + & + \\
\hline & & & 236 & - & - & + & + & + & + \\
\hline & & & 23 & - & - & - & + & + & + \\
\hline \multirow{2}{*}{$\mathbf{K} 2$} & & & 5 & - & - & - & $-*$ & + & + \\
\hline & & & 0.5 & - & - & - & - & - & - \\
\hline \multirow[t]{3}{*}{151} & \multirow{3}{*}{\multicolumn{2}{|c|}{ Plates contaminated }} & $38.5 \times 10^{5}$ & - & - & $t$ & + & + & + \\
\hline & & & $3 \cdot 8$ & - & - & + & $-*$ & $-*$ & + \\
\hline & & & $0 \cdot 3$ & - & - & - & $-^{*}$ & + & + \\
\hline \multirow[t]{3}{*}{170} & \multirow{3}{*}{\multicolumn{2}{|c|}{ Plates contaminated }} & $10 \times 10^{6}$ & - & $-^{*}$ & + & + & + & + \\
\hline & & & 10 & - & $-^{*}$ & + & + & + & + \\
\hline & & & 1 & - & - & - & + & + & + \\
\hline & - & 0.0089 & $7 \times 10^{6}$ & - & - & - & - & + & + \\
\hline \multirow[t]{3}{*}{ (repeated) } & & & 70 & - & - & - & - & + & + \\
\hline & & & 7 & - & - & - & - & + & + \\
\hline & & & 0.7 & - & - & - & - & + & + \\
\hline & - & - & $34 \times 10^{5}$ & - & - & + & $\div$ & + & + \\
\hline \multirow[t]{2}{*}{ (repeated) } & & & 3 & - & - & $-*$ & + & -* & - \\
\hline & & & 0.3 & - & - & - & $-*$ & $-^{*}$ & $-* *$ \\
\hline & - & - & $13 \times 10^{6}$ & - & + & + & + & + & + \\
\hline \multirow{2}{*}{ (repeated) } & & & $131 \cdot 5$ & - & + & + & + & + & + \\
\hline & & & 13 & - & $-^{*}$ & + & + & + & + \\
\hline
\end{tabular}

Inoculum size-gonococci per $10 \mathrm{ml}$ calculated using Miles and Misra counts

* Growth only after penicillinase was added

**The annomalous growth in a control after penicillinase was added

Table 3 Comparison of sensitivities arranged in rank order

\begin{tabular}{lll}
\hline $\begin{array}{l}\text { Sensitivities } \\
\text { (iu) }\end{array}$ & Plate method & Liquid medium \\
\hline $8 \cdot 5$ & 114 & \\
- & 97 & 112 \\
- & $\mathrm{V}$ & 97 \\
- & 167 & $\mathrm{~V}$ \\
- & 112 & 114 \\
- & $*$ & 167 \\
- & $*$ & 151 \\
- & $\mathrm{III}$ & 170 \\
- & $\mathrm{VII}$ & $\mathrm{III}$ \\
- & $\mathrm{K}_{2}$ & $\mathrm{VII}$ \\
0.0089 & 172 & 172 \\
\hline
\end{tabular}

*Plate values for 151 and 170 not available

used. The inhibition was much more marked when fresh guinea-pig complement was also present but not if reconstituted freeze-dried complement was used.

Serology in gonorrhoea is of limited value because reactions are often non-specific and there is a latent period between infection and a positive serological result. At present there appears to be no ideal method for the early detection of significant levels of antibody. This experiment shows that further study is needed into the possibility of using serum from patients to inhibit the growth of gonococci in liquid medium as an alternative to the methods currently available.

I am grateful to Difco for the studentship under which I was able to carry out this work. I am also grateful to Professor M. G. McEntegart, Dr S. Hafiz, and the staff of the Medical Microbiology Department, Sheffield University Medical School, Dr R. S. Morton of the Special Clinic, Royal Infirmary, Sheffield, and Dr A. E. Jephcott, Public Health Laboratory, Northern General Hospital, Sheffield, for providing the facilities for this work, and to Mrs H. Bland and Mrs H. Ashford for their help in typing the manuscript. 
Table 4 The inhibition of growth of gonococci in ANM liquid medium by incorporating hyperimmune serum and adding fresh complement

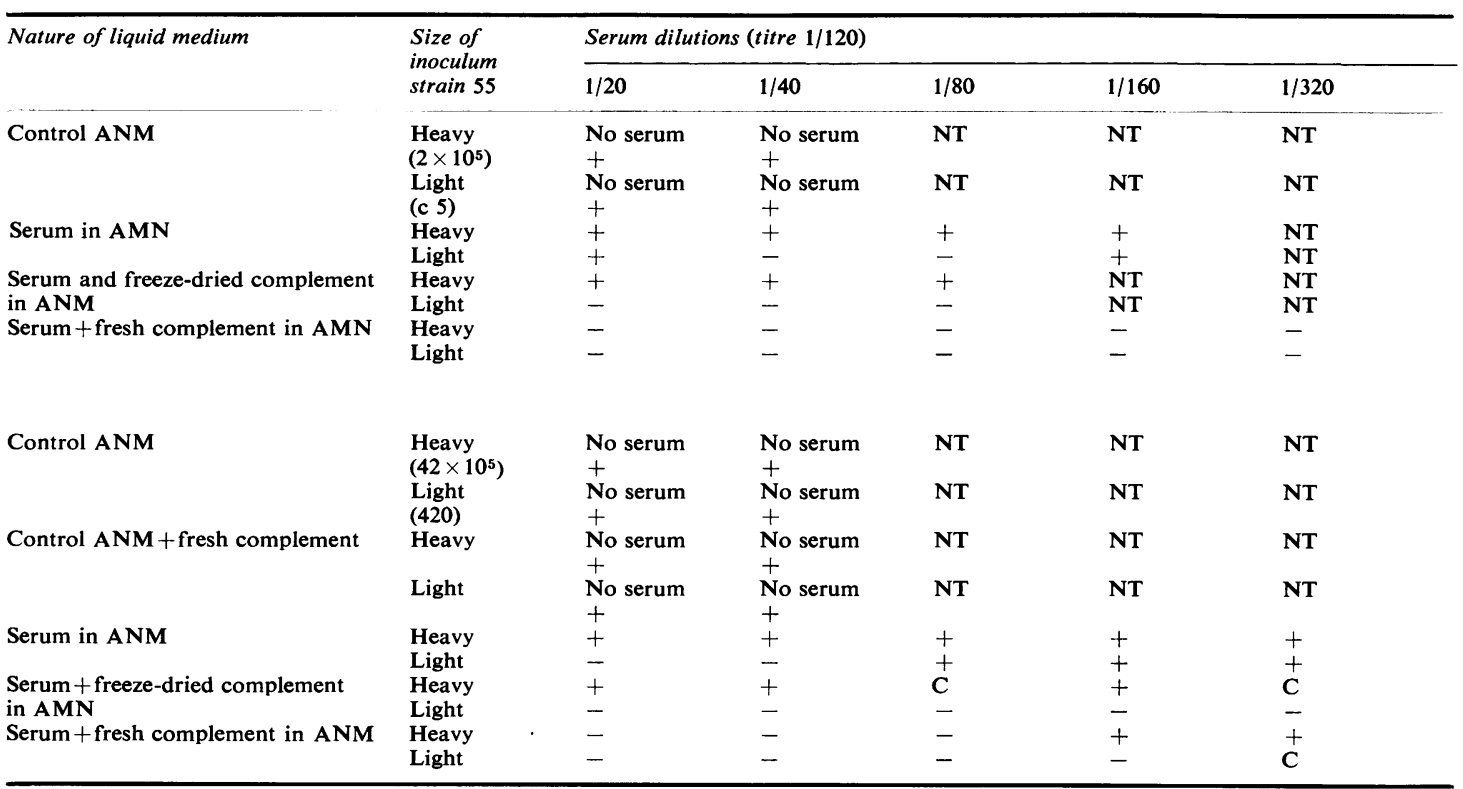

Source of fresh complement was millipore filtered fresh guinea-pig serum

$\mathrm{C}=$ Con1aminated

+ Growth on 24-hour subculture

- No griowth on 24-hour culture

$\mathbf{N T}=\mathbf{N o t}$ tested

\section{References}

Hafiz, S., and McEntegart, M. G. (1976). Prolonged survival of Neisseria gonorrhoeae in a new liquid medium. British Journal of Venereal Diseases, 52, 381-383.

Lawson, J. W., and Douglas, J. T. (1973). Induction and reversion of the L-form of Neisseria gonorrhoeae. Canadian Journal of Microbiology, 19, 1145-1151.

Miles, A. A., Misra, S. S., and Irwin, J. O. (1938). The estimation of bacteriocidal power of blood. Journal of Hygiene (London), 38, 732-748.
Reyn, A., and Bentzon, M. W. (1965). International Reference Strains of Neisseria gonorrhoeae for Use in Determination of Susceptibility to Penicillin. VDT/Res/Gon.9.65. WHO: Geneva.

Reyn, A., Bentzon, M. W., and Ericsson, H. (1963). Comparative investigations of the sensitivity of $N$. gonorrhoeae to penicillin. Acta pathologica et microbiologica Scandinavica, 57, 235-255.

Reyn, A., Korner, B., and Bentzon, M. W. (1958). Effects of penicillin, streptomycin, and tetracycline on $N$. gonorrhoeae isolated in 1944 and 1957. British Journal of Venereal Diseases, 34, 227-239. 